\title{
Improving laparoscopic skills using a novel pelvitrainer: A pilot study
}

\author{
Hany Mohamed El-Barbary, MD, FRCSI* \\ Department of General Surgery, Ain Shams University, Cairo, Egypt.
}

*(Presented in the 7th International Congress of the Egyptian Society of Laparoscopic Surgery [ESLS], in collaboration with Mediterranean \& Middle Eastern Endoscopic Surgery Association [MMESA], Jan 2009)

\begin{abstract}
Introduction: Simulators (Lap trainers) are essential for training surgeons on the basic and advanced laparoscopic skills. However, it is not available in most of our hospitals. The high cost and the difficulty in attending hands on training courses for the majority of surgeons in developing countries is reflected on the level of practice and on the patient's safety. In this study, there is an introduction and evaluation of a novel and cheap pelvitrainer, which could be an aid for training in laparoscopy in developing countries, as well as a brief account on the importance of having a structured training program.

Materials and methods: Thirty $(n=30)$ general surgical residents, years $1 \& 2$ in training, at Ain Shams University Hospital, Cairo, Egypt, were enrolled in a prospective, randomized, controlled, double blind study. In the first study group $(n=15)$, residents were randomly assigned to have one hour training using the new pelvitrainer for 3 consecutive days. The training exercises were composed of three levels of difficulty, starting with pick \& place exercise, then precision cutting and lastly laparoscopic suturing. Following this, their ability to perform efficient laparoscopic dissection of the gall bladder bed during laparoscopic cholecystectomy in the operating theater, under supervision, was assessed by an independent observer who was a surgery consultant. Following this, the trainees were given questionnaires to evaluate the process. On the other hand, concurrently, a comparable number of residents controls $(n=15)$, who have not been trained on the pelvitrainer, were also evaluated during performing the same operative procedure, under supervision. The results of the evaluations and questionnaires were subjected to statistical analysis.

Results: There was a statistically significant difference in the performance of surgical residents who have been trained on the new pelvitrainer in the study group and who successfully completed the three levels of pelvitrainer exercises compared to controls, which was shown by the time spent to perform the operative procedure and the number of mistakes. On the other hand, the degree of performance of the control and study groups together was also related to an independent factor which was the frequency of exposure to laparoscopic cholecystectomy. Residents in the study group showed increased interest in the new pelvitrainer.

Conclusion: The new pelvitrainer might be a useful and cheap adjunct to surgical resident training in our hospitals as well as others in developing countries.
\end{abstract}

Key words: Pelvitrainer - Laparoscopic training.

\section{Introduction:}

There are three levels of human behavior:

$>$ Skill-based: learned by training \& experience (example: suturing).
$>$ Rule-based: in which action is triggered by a stored rule (example: recognizing anatomy and pathological alterations).

$>$ Knowledge-based: in which action is based on analysis \& overall aim (example: management of intraoperative bleeding). 
Simulators (Lap trainers) are very essential for training of surgeons on the basic and advanced laparoscopic skills. Pelvitrainer exercises are focused on the skill-based learning of tasks as laparoscopic suturing. Rule-based behavior is acquired through lectures, textbooks and video instructions. Furthermore, in the OR, most of the knowledge-based behavior necessary to deal with complications and emergencies will be acquired. A living animal model provides the only way to acquire this knowledge outside the operating room; however it is discouraged by some government regulations in some countries. ${ }^{1}$

\section{The problem:}

1- Pelvitrainers are not available in most of our hospitals.

2- High cost of pelvitrainers.(EGP1000-6000) Figure(1).

3- Lack of a structured training program.

4- Limited hands-on courses.

Many sophisticated and expensive trainers have been developed to assist surgeons in learning basic laparoscopic skills. We developed an inexpensive trainer and evaluated its effectiveness.

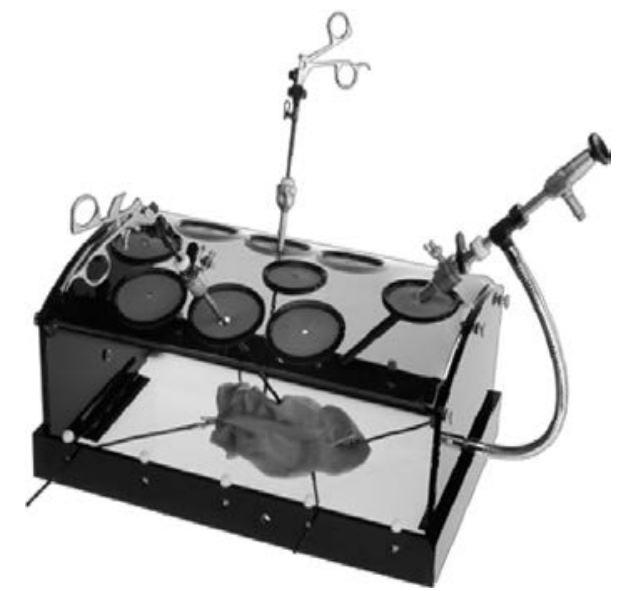

Figure (1): The Szabo-Berci pelvitrainer.

\section{The innovation:}

"Homemade multipurpose Lap-Trainer with integrated Web camera \& simple light source" Figure(2) Patent No: 1978/2008 in 4/12/2008, Academy of Scientific Research and Technology, Egyptian Patent Office. Setup:

Comprises the training box with an integrated high resolution webcam and a computer. The picture is real time, obtained through a pre-installed software. Training can be done using synthetic or organic models with the available disposable laparoscopic instruments.

\section{Savings:}

$>$ No limited day's courses.

$>$ No need for laparoscope $(\mathrm{EGP}=120$ trainer vs. $E G P=120,000$ laparoscope).

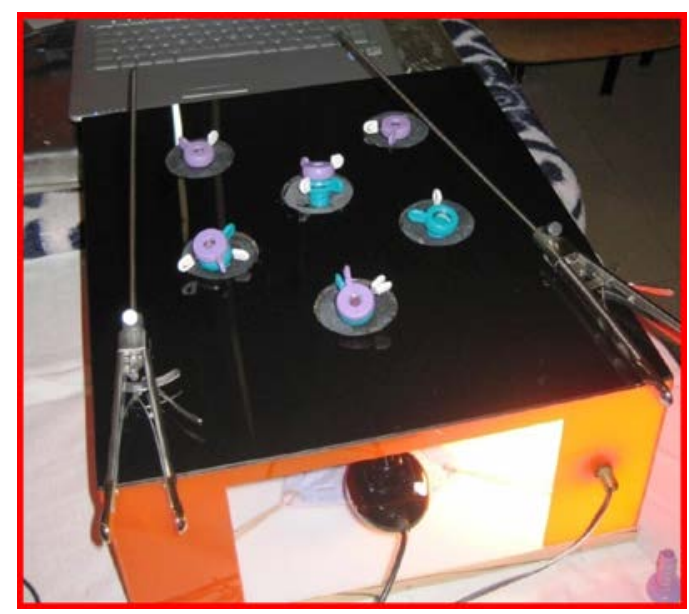

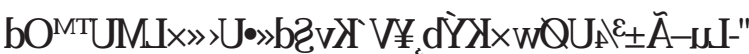
$\left[0^{\circ} w s \omega\right\}, d q \pm \exists \vec{\exists} W d \pm d \pm \gg b O \pm U l^{\circ}$ "ÂUИ $\rfloor x » s 0 d \exists \grave{Y} \pm\left|M g x » w X_{A}\right|_{ \pm}$

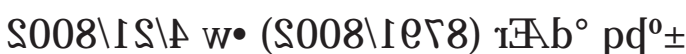

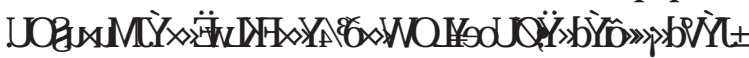

Figure (2): The novel pelvitrainer (patent no.:1978/2008). 


\section{New aspect of this patent:}

1- Simple \& cost effective design.

2- All materials are available as cast acrylic sheets from which it is made. The integrated camera is a 2 megapixel webcam.

3- Multipurpose functionality as it can be used either as office based with a Laptop or in a workshop with a real laparoscope.

4- Cheap, as it costs less than EGP 200 excluding the Web Cam \& the Laptop (about Eur. 25).

\section{Aim of the study:}

Evaluation and validation of the new pelvitrainer.

\section{Study design:}

Prospective, randomized, controlled, double blind study.

\section{Materials and methods:}

Thirty $(n=30)$ general surgical residents, years $1 \& 2$ in training, at Ain Shams University Hospital, Cairo, Egypt, were enrolled in a prospective, randomized, controlled, double blind study. In the first study group $(n=15)$, residents were randomly assigned to have one hour training using the new pelvitrainer for 3 consecutive days. Figure(3) The training exercises were composed of three levels of difficulty and each rated 1-5, starting with pick $\&$ place exercise (test 1 ), then precision cutting (test 2) and lastly laparoscopic suturing (test3). Following this, their ability to perform efficient laparoscopic dissection of the gall bladder bed during laparoscopic cholecystectomy in the operating theater under supervision, was assessed by an independent observer who was a surgery consultant. Following this, the trainees were given questionnaires to evaluate the process. On the other hand, concurrently, a comparable number of residents controls $(n=15)$, who have not been trained on the pelvitrainer, were also evaluated during performing the same operative procedure, under supervision. The trainees in the study group answered questionnaires (for validity) and a performance checklist was filled up by an independent observer (for proficiency) for both groups during performing supervised laparoscopic gall bladder dissection.

This objective assessment was done for all participants as follows:

\section{Validity Questionnaires:}

(For study group; tick one choice)

1- The possibility to train using this pelvitrainer?

Useless

Reasonable

Usefull

Good

Excellent

2- The time given to train using this pelvitrainer? (1HR):

Too Short

Long Enough

Too Long

3- If available in your hospital, how many HRS/W would you need to train using this pelvitrainer?

$1 \mathrm{HR}$

2-5 HRs

$>5$ HRs

4- Should training on pelvitrainers become part of your final assessment?

YES

NO

DN

5- Should training on pelvitrainers become required before operating on patients? YES

NO

DN

\section{Proficiency Checklist:}

(For study \& controls)

1- RATED 1-5; involved assessment of:

Precision, coordination, orientation, handling and force / tension.

2- Time in minutes.

All results were analyzed using the SPSS 17.0 software. 


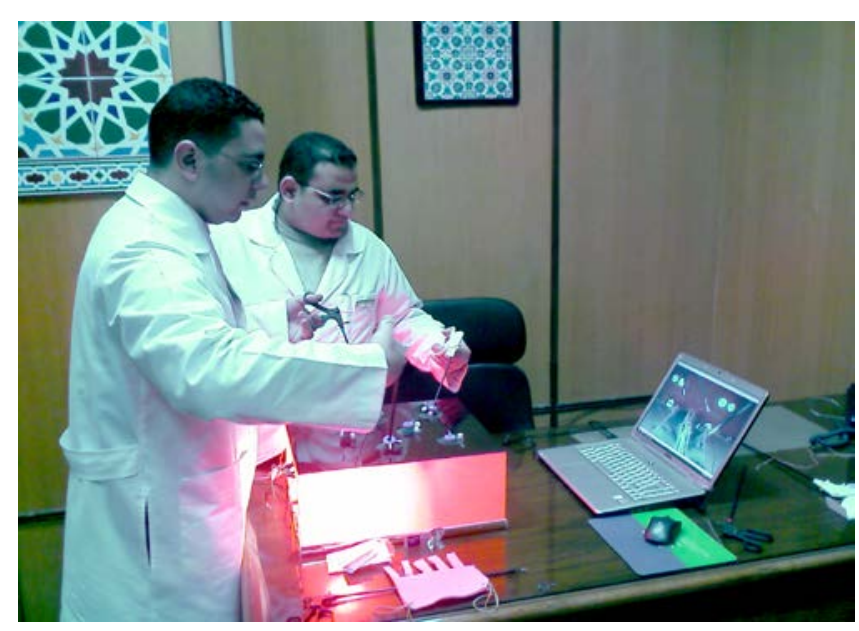

Figure (3): Practicing on the new pelvitrainer.

\section{Results:}

There was a statistically highly significant difference in the performance of surgical residents (proficiency) in the operating theater (OR), who have been trained on the new pelvitrainer in the study group and who successfully completed the three levels of pelvitrainer exercises (rated 1-5 each), compared to controls Figure $(\mathbf{5 , 6})$, which was shown by the time spent to perform the operative procedure and the number of mistakes i.e.: Proficiency in the OR (rated 1-5)

Table(1,2).

Table (1): Descriptive data of both groups.

\begin{tabular}{|c|c|c|c|c|c|c|}
\hline Study & Test 1 & Test 2 & Test 3 & Average & Proficiency & Time \\
\hline Mean & 4.1 & 3.8 & 3.1 & 3.7 & 3.7 & $13 \mathrm{~min}$ \\
\hline Std. d & 0.8 & 1.1 & 1.0 & 0.7 & 0.9 & 2.7 \\
\hline $\begin{array}{l}\text { Contro } \\
\mathrm{n}=15 \\
\text { Mean }\end{array}$ & & & & & 2.4 & $18 \mathrm{~min}$ \\
\hline Std. d & & & & & 1.2 & 6.2 \\
\hline
\end{tabular}

Table (2): Comparison between study \& controls as regards proficiency \& time in $O R$. HS = highly significant.

\begin{tabular}{|l|c|c|c|c|c|c|}
\hline \multirow{2}{*}{ Proficiency } & Code & Number & Mean & Std. d & T-test & Significance \\
\cline { 2 - 6 } & Study & 15 & 3.7 & 0.9 & 3.5 & \multirow{2}{*}{$0.002 \mathrm{HS}$} \\
\cline { 2 - 6 } & Controls & 15 & 2.4 & 1.2 & & \\
\hline \multirow{2}{*}{ Time } & Study & 15 & 13 & 2.7 & -2.8 & \multirow{2}{*}{$0.009 \mathrm{HS}$} \\
\cline { 2 - 5 } & Controls & 15 & 18 & 6.2 & & \\
\hline
\end{tabular}




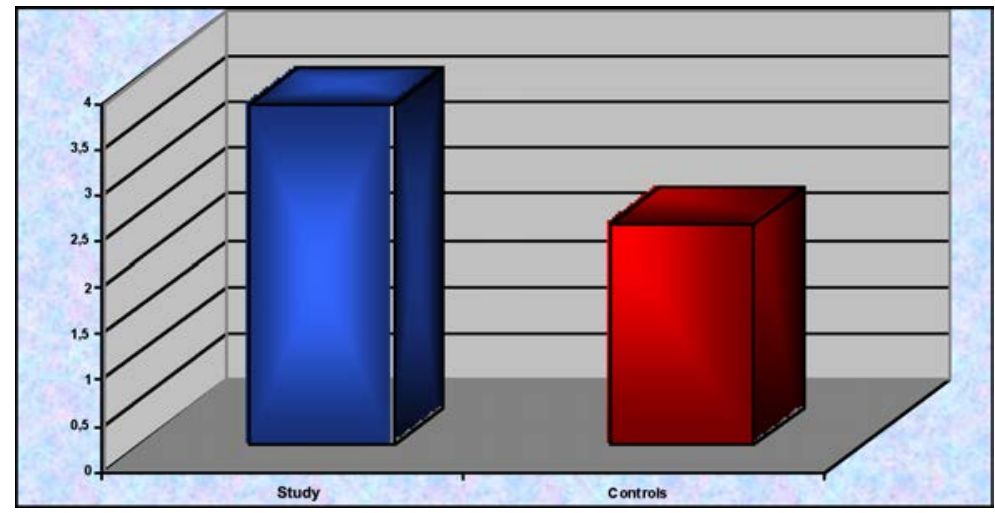

Figure (4): Comparison between studied residents and controls as regards Proficiency.

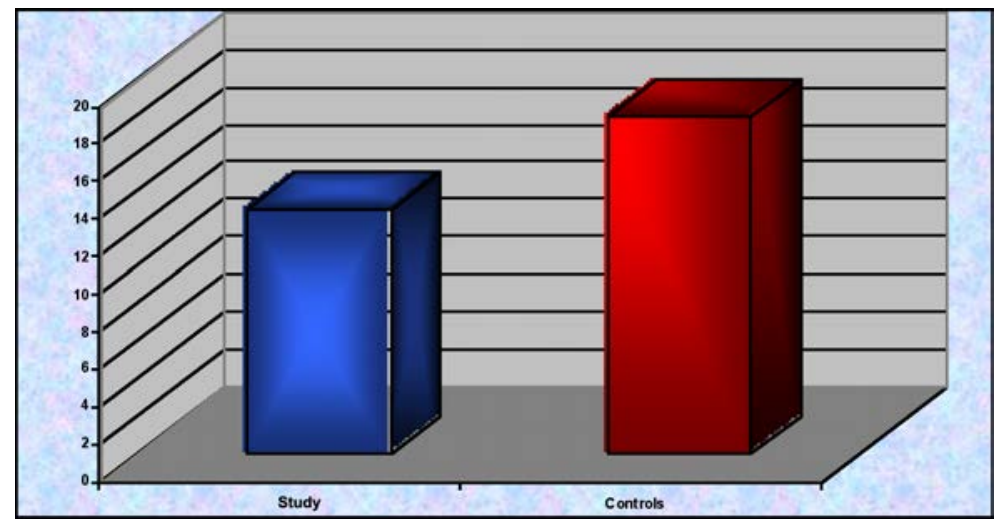

Figure (5): Comparison between studied residents and controls as regards time.

\section{Results:}

The average time spent to dissect the gall bladder bed in the study group was 13 minutes, whereas in the controls who were not trained

on the pelvitrainer was 18 minutes. The graph in Figure(6) shows the correlation between proficiency \& time.

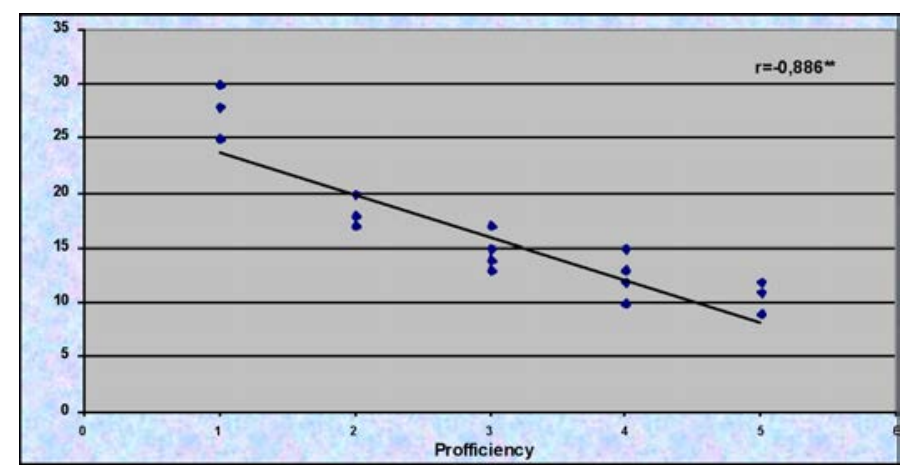

Figure (6): Correlation between proficiency and time in OR in both groups.

As regards validity, residents in the study group showed increased interest in the new pelvitrainer; $7 \%$ found it useful, $40 \%$ good \& $53 \%$ found it excellent. All residents (100\%) agreed that training on pelvitrainers should become required before operating on patients.
Sixty seven percent of studied residents found training on the pelvitrainer for one hour per day enough. Fifty three percent stated that they need $2-5 \mathrm{hrs} / \mathrm{w}, 47 \%$ will need $>5 \mathrm{hrs}$ and $73 \%$ thought that pelvitrainer exercises should be part of their final assessment. 


\section{Discussion:}

Training on skill-based behavior is encouraged as laparoscopy combines unusual hand-eye coordination with the use of complex instruments; this is usually teached in a 2-3 day introductory course. Instrument $\&$ tissue handling as well as lap suturing are trained. ${ }^{2}$

The benefit of having available a simple pelvitrainer was apparent in this study. Residents who practiced training prior to applying the acquired skills on humans surpassed their counterparts in the control group in proficiency, with a mean score of 3.7 for the former compared to 2.4 for the latter. On the other hand, the time of performance of the operative procedure in the study and control groups together was relatively long, although shorter in the study group (13 min in study compared to $18 \mathrm{~min}$ in controls). This may be related to an independent factor which was the frequency of exposure to laparoscopic cholecystectomy i.e. previous experience. For the safety of our patients, the training objectives should be defined. 1,3 This is represented by the level of competence acquired after training in a residency program. Fulfilling all training needs by only one method will require a highly complex and very expensive trainer which is yet not available. The complexity and cost of a training means are relatively low if the training objective comprises skill-based behavior only, since this can be trained with simple pelvitrainers. ${ }^{1}$

The cost of this new pelvitrainer is incomparable to others i.e. EGP 200 $=$ EURO 25 , which is less than the cheapest homemade training box..$^{4,5}$

For junior laparoscopic surgeon, the webcam system is an inexpensive and effective laparoscopic training device. Furthermore, the webcam system also allows instant recording and review of techniques. ${ }^{6}$

The ideal training for a laparoscopic procedure is, firstly, for trainees to watch a video; if they have no laparoscopic experience, they should spend time on a pelvitrainer before attending a hands-on course. The trainee should then arrange for an experienced surgeon to act as a proctor at his or her hospital. ${ }^{7}$ The study group agreed upon the importance of having pelvitrainers available in their institute in conjunction with proctor supervision. The need for a structured and validated training program in laparoscopy could not be overemphasized. ${ }^{8}$

\section{Conclusion:}

Many sophisticated and expensive trainers have been developed to assist surgeons in learning basic laparoscopic skills. The new pelvitrainer might be a useful and cheap adjunct to surgical resident training in our hospitals as well as others in developing countries.

\section{References:}

1- Dankelman J: Surgical Simulator Design and Development. World J Surg 2008; 32(2): 149-155.

2- Mishra R: Role of training in minimal access surgery. In: Text book of practical laparoscopic surgery 2007; p.411-429. Jaypee.

3- Rosenthal R, Gantert W, Hamel C, et al: The future of patient safety: Surgical trainees accept virtual reality as a new training tool. Patient Saf Surg 2008; 2: 16.

4- Ricchiuti D, Ralat D, Evancho-Chapman $\mathrm{M}$, et al: A simple cost-effective design for construction of a laparoscopic trainer. Journal of Endourology 2005; 19(8): 10001005.

5- Huri E: Poor man's laparoscopy: A simple low cost laparoscopic box trainer using a 5 megapixel web camera: Poster Session, 2nd prize winner, Karl Storz Awards, EAU 2nd North Eastern European Meeting, Vilnius, Lithuania, 12-13 September 2008.

6- Chung D, Landsittel C, Chon C, et al: Laparoscopic skills training using a webcam training. The Journal of Urology; 173: 180183.

7- Royston C, Lansdown M, Brough W : Education and debate: Teaching laparoscopic surgery; the need for guidelines. BMJ 1994; 308: 1023-1025.

8- Stolzenburg J, Truss M, Rabenalt, et al: Training in laparoscopy. EAU-EBU update series 2007; 5: 53-62. 